\title{
EFEITOS DO FITOTERÁPICO PHASEOLUS VULGARIS L. SOBRE A SACIEDADE DURANTE O TRATAMENTO DA OBESIDADE
}

\section{EFFECTS OF PHYTOTHERAPY PHASEOLUS VULGARIS L. ON SATIETY DURING THE TREATMENT OF OBESITY}

\author{
Maria Clara Francisco Nunes ${ }^{1}$, Marclênia Eduardo Ramos ${ }^{2}$, Erika Cardoso Abud Parreira ${ }^{3}$ \\ Universidade Federal de Viçosa ${ }^{1}$; Centro Universitário do Triângulo²; Faculdade Presidente Antônio Carlos ${ }^{3}$
}

\begin{abstract}
Obesity is a worldwide health problem, therefore there has been an expansion of treatments for the same, among which the diet control, together with the use of herbal medicines, stands out. Phaseolus vulgaris L. (Beans) properties were reviewed in indexed journals to evaluate their efficacy on satiety and consequent weight loss in obese individuals. For the composition of the study, the descriptors in Portuguese and English were consulted through the virtual library Descriptors of Health Science (DeCS) and searches in PubMed, SciELO and LILACS databases. The literature indicates a positive effect of the active substance of Phaseolus vulgaris $L$. on satiety and weight loss. Thus, phaseolamine can be considered an adjuvant to the treatment of obesity, but new clinical studies are necessary to prove its efficacy.
\end{abstract}

Key words: obesity, phytoterapeutic drugs, satiety response, weight loss.

\section{Resumo}

A obesidade é um problema mundial de saúde, em consequência houve uma expansão de tratamentos para o mesmo, dentre os quais se destaca o controle da dieta, aliado ao uso de medicamentos fitoterápicos. Foi realizada revisão, em periódicos indexados sobre as propriedades da Phaseolus vulgaris L. (Feijão), objetivando avaliar a sua eficácia sobre a saciedade $e$ consequente perda de peso em indivíduos obesos. Para a composição do estudo, fora realizada consulta aos descritores em língua portuguesa $e$ inglesa através da biblioteca virtual Descritores em Ciência da Saúde (DeCS) e buscas nas bases de dados PubMed, SciELO e LILACS. A literatura aponta um efeito positivo da substancia ativa de Phaseolus vulgaris $L$. sobre a saciedade e perda de peso. Dessa forma, pode ser considerada um adjuvante ao tratamento da obesidade, porém são necessários novos estudos clínicos a fim de comprovar a sua eficiência.

Palavras-chave: obesidade, medicamentos fitoterápicos, saciedade, perda de peso. 


\section{Introdução}

A obesidade caracteriza-se pelo acúmulo excessivo de gordura corporal, que pode prejudicar a saúde, em decorrência de hábitos alimentares inadequados e sedentarismo. Além disso, fatores genéticos, ambientais e sociais, também são aspectos relevantes que devem ser considerados como causas desse problema mundial de saúde ${ }^{1}$.

Segundo dados da World Health Organization (WHO), cerca de $13 \%$ dos adultos com idade igual ou superior a 18 anos, eram obesos, em todo o mundo no ano de $2014^{1}$. A prevalência da obesidade que antes estava associada apenas a países desenvolvidos, atualmente prevalece também em países em desenvolvimento e subdesenvolvidos, principalmente em grandes centros urbanos, provocando a morte de 2,8 milhões de pessoas a cada ano, em todo o mundo 2 .

De acordo com o Mapa da Obesidade desenvolvido pela Associação para o Estudo da Obesidade e da Síndrome Metabólica (ABESO), com base em dados da Pesquisa de Orçamentos Familiares (POF) realizada entre 2008 e 2009, cerca de $50 \%$ da população brasileira adulta se encontra na faixa de sobrepeso ou obesidade, sendo as regiões de maior incidência, as regiões Sul $(56,08 \%)$ e Sudeste $(50,45 \%)^{3}$.

Com o aumento da incidência do excesso de peso e obesidade, houve uma expansão da oferta de tratamentos na área de emagrecimento que, normalmente se caracteriza inicialmente pela diminuição da ingestão calórica diária, aumento da prática de atividades físicas e modificação de hábitos de vida. Porém, quando não se consegue os objetivos desejados, outros recursos são procurados, como a utilização de medicamentos alopáticos e, por fim, as cirurgias. No entanto, estes últimos recursos apresentam alto custo e podem gerar graves efeitos colaterais ${ }^{4,5}$.

Dessa forma, se faz necessária a utilização de recursos mais acessíveis, como as práticas integrativas e complementares, que foram incluídas no Sistema Único de Saúde (SUS), no Brasil, através da Política Nacional de Práticas Integrativas e Complementares (PNPIC), dentre as quais se destaca o uso de plantas medicinais $\mathrm{e}$ a fitoterapia, que se caracterizam como opções no tratamento da obesidade ${ }^{6,7,8}$.

A fitoterapia corresponde ao tratamento terapêutico com base no uso de plantas medicinais ou partes da mesma, em suas diferentes formas farmacêuticas, que não apresentam substâncias ativas isoladas, mesmo que seja de origem vegetal ${ }^{6}$. Segundo a Pesquisa Nacional de Saúde realizada em 2013, cerca de $3,8 \%$ da população, utilizaram alguma prática integrativa e complementar, como o emprego de plantas medicinais e fitoterapia, sendo essa prática mais comum nas Regiões Norte $(5,9 \%)$ e Sul $(5,2 \%)^{9}$.

Dentre os fitoterápicos utilizados no tratamento da obesidade se destacam os moderadores da saciedade, como a espécie Phaseolus vulgaris $L$. (Feijão) ${ }^{10}$.

O controle da saciedade pode ser utilizado como estratégia durante 0 tratamento da obesidade, visto que as pessoas vivem constantemente sob a exposição de alimentos calóricos, relativamente baratos ${ }^{11,12}$. A saciedade corresponde ao estado no qual o indivíduo se sente satisfeito depois de alimentar-se e utiliza essa percepção para controlar a ingestão de alimentos $^{13}$. Dessa forma a moderação da saciedade tem grande importância sobre a incidência mundial de sobrepeso e obesidade e no controle do consumo energético excessivo ${ }^{14}$.

Diante do exposto, o objetivo deste estudo foi avaliar através de uma revisão literária a eficácia das propriedades da Phaseolus vulgaris $L$. sobre a saciedade e consequente perda de peso em indivíduos obesos, durante o tratamento da obesidade.

\section{Materiais e Métodos}

Foi realizada revisão, em periódicos indexados sobre as propriedades da Phaseolus vulgaris $L$. especificamente em relação ao aumento da saciedade e consequente perda de peso, no tratamento da obesidade. Para atingir o objetivo do estudo, as buscas foram realizadas nas bases de dados PubMed, SciELO e LILACS, sendo incluídos trabalhos publicados entre 2003 e 2017.

A consulta aos descritores foi realizada em língua portuguesa e inglesa através da biblioteca virtual Descritores em Ciência da Saúde (DeCS).

Os descritores utilizados foram Phaseolus vulgaris L., phytotherapeutic drug, obesity, weight loss, satiety response, combinados entre si, bem como seus respectivos em português: Phaseolus vulgaris L., medicamentos 
fitoterápicos, obesidade, perda de peso, saciedade.

Foram encontrados 70 artigos referentes ao tema, tendo sido realizada a leitura dos títulos e resumos aplicando os seguintes critérios de inclusão: estudos com delineamento experimental ou observacional, resultados de perda de peso ou não, aumento ou não da saciedade e uso de Phaseolus vulgaris L.. No total 32 artigos cumpriram os critérios de inclusão, tendo sido lidos na íntegra e analisados, dos quais foram coletadas informações para alcançar o objetivo dessa revisão.

A exposição dos resultados e a discussão dos dados obtidos é realizada de forma descritiva, possibilitando ao leitor a avaliação da aplicabilidade da revisão desenvolvida.

\section{Resultados e Discussão}

Esta planta citada neste artigo pode ser prescrita pelo nutricionista, segundo a lista estabelecida na Instrução Normativa no 2, de 2014, da ANVISA 15, além das descritas no Parecer CRN 3 (29/07/2010) e das drogas vegetais listadas na RDC $n^{\circ} 10$ de 201016. Porém, é necessário ter especialização na área fitoterápica para realizar a prescrição, cuja regulamentação se dá pela Resolução CFN N ${ }^{\circ}$ $525 / 2013$

\section{Phaseolus vulgaris $L$}

\section{Características da planta}

Caracteriza-se por se uma planta nutracêutica, herbácea pertencente à família Leguminosae, subfamília Faboideae, gênero Phaseolus, sendo classificada como Phaseolus vulgaris L.. Existem em torno de 50 variedades nativas de Phaseolus vulgaris $L$. na América Latina, sendo uma planta típica de áreas de clima temperado $17,18,19$.

O feijão (Phaseolus vulgaris L.) corresponde a um dos alimentos básicos consumidos pela população em todo o mundo. Constitui-se em uma das principais fontes de proteínas, carboidratos, minerais, vitaminas e fibras, além de conter variedade de compostos fotoquímicos com propriedades benéficas para a saúde, contribuindo no tratamento de doenças crônicas degenerativas, como a obesidade ${ }^{20,18}$.

No entanto, possui em sua composição, fatores anti-nutricionais, como os fitatos que prejudicam a absorção de outros micronutrientes. Apesar disso, os carboidratos são os principais componentes nutricionais do feijão, correspondendo a cerca de $50 \%$ da sua matéria seca, sendo rico em amido lentamente digerível, o chamado amido resistente $e^{20,18,21}$.

O feijão apresenta um teor total de proteína que varia de $16 \%$ a $33 \%$, na sua composição, sendo elas de baixo valor biológico. As principais proteínas encontradas nos grãos do gênero Phaseolus são as globulinas e as albuminas, que são encontradas em maior quantidade e as prolaminas e glutelinas que são encontradas em menor quantidade. A globulina com maior concentração é faseolamina, cujo conteúdo corresponde a cerca de $50 \%$ do total de globulinas presentes no feijão ${ }^{18,22,23}$.

\section{Mecanismo de ação}

A saciedade é caracterizada pela percepção da fome, seguida de eventos metabólicos e resposta cerebral ao processo, assim sinais fisiológicos são acionados em resposta a ingestão de alimentos, mantendo uma inibição sobre comer por um determinado período ${ }^{13}$.

A justificativa utilizada para a utilização da faseolamina no auxílio da perda de peso é que ela provoca bloqueio da $\alpha$-amilase (enzima digestiva de carboidratos) provocando alterações no processo de resposta glicêmica, reduzindo os níveis de glicemia pós-prandial e consequentemente aumento no tempo do esvaziamento gástrico colaborando para o modulação e aumento da saciedade 24,25,18.

A enzima $\alpha$-amilase presente na saliva e em secreções pancreáticas, responsável pela hidrólise de amido presente em carboidratos, pode ser modulada afetando a utilização do mesmo como fonte de energia, devido ao surgimento de substâncias de origem microbiana ou de tecidos vegetais, como a faseolamina presente no Phaseolus vulgaris $L$. (feijão comum), principalmente no feijão branco ${ }^{26}$.

Essa substância conhecida por ter efeito anti-obesidade, apresenta atividade inibitória efetiva da enzima $\alpha$-amilase em todo o trato gastrointestinal de organismos in vivo, durante o processo de degradação de carboidratos. A ação da faseolamina retarda a digestão dos mesmos e reduz a taxa de absorção de glicose no intestino e a glicemia pós-prandial, posteriormente ${ }^{27}$. 
Quando administrada antes ou durante das refeições, a faseolamina possibilita o controle dos níveis de glicose sanguínea e a diminuição da taxa de absorção corporal de energia em forma de glicose ${ }^{27}$.

A faseolamina presente na planta Phaseolus vulgaris L. bloqueia a atividade enzimática da $\alpha$ amilase, pois caracteriza-se como uma glicoproteína, extraída do feijão branco, de estrutura rígida e compacta com baixa atividade hidrofílica que limita a acessibilidade das enzimas proteolíticas à sua estrutura, tornando-a altamente resistente à digestão ${ }^{28,22}$.

Existem fatores funcionais e bioquímicos envolvidos no mecanismo de ação da faseolamina e os efeitos destes sobre ela, que ainda precisam ser estudados e compreendidos. Uma das explicações sobre a interferência da faseolamina na digestão e absorção do amido, é que essa ação ocorre através da inibição completa do acesso do mesmo ao sítio ativo da enzima $\alpha$-amilase. Durante o processo, a substância bloqueadora, liga-se ao sítio ativo da enzima $\alpha$-amilase por meio de alças formando uma rede de ligações, assim a enzima modifica sua estrutura e liga-se à substância inibidora ${ }^{27}$.

Assim a perda de peso aconteceria em decorrência da mobilização de reservas de gordura corporal devido à restrição de energia provinda de carboidratos, como resultado da ação inibidora da $\alpha$-amilase, provocada pela faseolamina ${ }^{27}$.

\section{Estudos realizados}

Uma entrevista realizada por 29, feita com 15 nutricionista de um município do Paraná, como o objetivo de conhecer quais os fitoterápicos mais utilizados entre os profissionais, a faseolamina foi citada como fitoterápico importante no auxílio da perda de peso.

Alguns estudos realizados relatam a ação de extratos secos de Phaseolus vulgaris $L$. sobre o metabolismo de carboidratos e modulação da saciedade em humanos.

Com o objetivo de avaliar o efeito de um extrato seco de Phaseolus vulgaris L. sobre o metabolismo de carboidratos e saciedade, uma pesquisa realizada por Spadafranca e colaboradores em $2012^{30}$, utilizou $100 \mathrm{mg}$ de extrato seco administrado em uma refeição normocalórica sem restrição, durante 1 semana.
A pesquisa contou com 12 indivíduos obesos, sendo controlada por placebo. A saciedade fora avaliada no início e durante três horas após a refeição. O estudo mostrou que durante essas três horas o extrato seco induziu a um desejo reduzido de comer entre os indivíduos, suprimindo a secreção de grelina (hormônio que induz a fome).

Um estudo conduzido por 25 , durante 4 semanas, com 59 voluntários diagnosticados com excesso de peso, objetivou avaliar a ação inibitória de extrato seco de Phaseolus vulgaris L. (feijão branco), sobre a enzima digestiva $\alpha$ amilase. Durante o período do estudo, o extrato seco foi ofertado em forma de comprimido de $800 \mathrm{mg}$ antes de uma grande refeição rica em carboidratos, com dieta de 2.000 a $2.020 \mathrm{Kcal}$ por dia. Os resultados dessa pesquisa mostraram redução significativa do peso corporal, IMC, massa gorda, e circunferências nos indivíduos que receberam o extrato da planta quando comparados ao grupo de indivíduos que receberam placebo.

Um estudo realizado por 30, durante 8 semanas a fim de avaliar os efeitos de um extrato seco de Phaseolus vulgaris L. (feijão branco), na perda de peso 27 participantes receberam durante esse período $1.500 \mathrm{mg}$ do extrato ou um placebo duas vezes ao dia, juntamente com almoço e jantar, sendo a dieta normocalórica e rica em fibras. Ao final da pesquisa não foi observado uma redução significativa do peso, porém houve redução dos níveis triglicerídes.

Uma pesquisa examinou um suplemento derivado de Phaseolus vulgaris L., contendo $1.000 \mathrm{mg}$ de extrato seco da planta. O estudo controlado por placebo contou com 101 voluntários com excesso de peso. O extrato foi ofertado em cápsulas 15 minutos após as refeições durante 2 meses. Depois desse períodos, os voluntários que receberam extrato seco apresentaram perda significativa de peso e circunferência da cintura, quando comparado ao placebo ${ }^{31}$.

De acordo com estudos realizados o extrato de Phaseolus vulgaris L., em nível toxicológico foi considerado seguro, mas ainda é necessário regulamentar a indicação do extrato da planta como estratégia utilizada em tratamentos para redução de peso. Existe a necessidade de realizar pesquisas maiores e com mais rigor sobre a ação eficaz do feijão branco no tratamento da 
obesidade $32,25,33,30$.

Efeitos adversos como flatulências, desconfortos intestinais e cólicas abdominais podem ser decorrentes do uso da faseolamina. 0 seu uso concomitante à agentes hipoglicemiantes não é recomendado pois pode aumentar seu efeito. Para indivíduos hipoglicêmicos e gestantes, o uso da faseolamina é contraindicada ${ }^{34}$.

\section{Conclusão}

A faseolamina, substância ativa da espécie Phaseolus vulgaris L. (feijão branco) pode ser considerada como um adjuvante promissor ao tratamento da obesidade, pois é um potencial redutor da absorção de carboidratos da dieta, devido a sua ação inibitória da enzima $\alpha$-amilase.

Apesar de ser considerado seguro em alguns estudos desenvolvidos, o uso da espécie Phaseolus vulgaris L. (feijão branco), deve ser moderado. São necessários estudos clínicos mais rigorosos a fim de comprovar a eficiência da planta, uma vez que existem poucos trabalhos referentes a ingestão em longo prazo de Phaseolus vulgaris $L$. e quais mecanismos de ação envolvidos no processo de modulação da saciedade e perda de peso.

\section{Referências}

1. World Health Organization. Obesity and overweight. 2014. Disponível em: http://www.who.int/mediacentre/factsheets/fs3 11/en/. Acesso em: 30 jul 2017.

2. World Health Organization. 10 facts on obesity. 2017. Disponível em: Acesso em: 30 jul 2017.

3. Associação Brasileira para o Estudo da Obesidade e da Síndrome Metabólica. Mapa da Obesidade. Disponível em: http://www.abeso.org.br/atitude-

saudavel/mapa-obesidade. Acesso em: 30 Junho 2017.

4. Ferreira M. A fitoterapia no combate à obesidade. Monografia (Mestrado em Ciências Farmacêuticas) 124f. Faculdade de Ciências e Tecnologias, Universidade do Algarve, Portugal, 2013.

5. De Freitas Junior L. M.; De Almeida Jr E. B. Medicinal plants for the treatment of obesity: ethnopharmacological approach and chemical and biological studies. American Journal of Translational Research, v. 9, n. 5, p. 2050-2064, 15 maio 2017.

6. Brasil. Ministério da Saúde, Portaria no 971 de 03 de maio de 2006. Aprova a Política Nacional de Práticas Integrativas e Complementares (PNPIC) no Sistema Único de Saúde. Gabinete do Ministro, Brasília, DF.

7. Yun J. W. Possible anti-obesity therapeutics from nature - A review. Phytochemistry, v. 71, n. 14, p. 1625-1641, 1 out. 2010.

8. Rodrigues A. G., De Simoni C. Plantas medicinais no contexto de políticas públicas. Informe Agropecuário, Belo Horizonte, v. 31, n. 255, p. 7-12, 2010.

9. Instituto Brasileiro de Geografia e Estatística. Pesquisa nacional de saúde 2013: acesso e utilização dos serviços de saúde, acidentes e violências: Brasil, grandes regiões e unidades da federação. 2013. 100 p, Rio de Janeiro, RJ.

10. Astell K. J.; Mathai M. L.; Su X. Q. A review on botanical species and chemical compounds with appetite suppressing properties for body weight control. Plant Foods for Human Nutrition (Dordrecht, Netherlands), v. 68, n. 3, p. 213-221, set. 2013.

11. Gerstein D.E., Woodward- Lopez G., Evans A., Kelsey K., Drewnowski A. Research and Professional Briefs: clarifying Concepts about Macronutrients' Effects on Satiation and Satiety. Journal of the American Dietetic Association, v.104, n. 7, p. 1151-1153, jul. 2004.

12. Solah V. A. et al. Effect of training on the reliability of satiety evaluation and use of trained panellists to determine the satiety effect of dietary fibre: A Randomised controlled trial. Plos One, v. 10, n.5, 15 may 2015.

13. Bornet F. R. J., Jardy-Gennetier A-E., Jacquet N., Stowell J. Glycaemic response to foods: Impact on satiety and long-term weight regulation. Appetite, v. 49, p. 535-553, 20 april, 2007.

14. Van Kleef E. , Van Trijp J. C. M , Van Den Borne J. J. G. C, Zondervan C. Successful development of satiety enhancing food products: Towards a multidisciplinary agenda of research challenges. Critical Reviews in Food Science and Nutrition, v. 52, p. 611-628, 2012.

15. Brasil. Agência Nacional de Vigilância Sanitária. Instrução Normativa $n^{\circ} 02$ de 13 de 
maio de 2014. Lista de medicamentos fitoterápicos de registro simplificado" e a "Lista de produtos tradicionais fitoterápicos de registro simplificado. Disponível em: http://bvsms.saude.gov.br/bvs/saudelegis/anvisa /2014/int0002_13_05_2014.pdf.

16. Brasil. Agência Nacional de Vigilância Sanitária. Resolução - RDC no 10, de 9 de março de 2010. Dispõe sobre a notificação de drogas vegetais junto à Agência Nacional de Vigilância Sanitária (ANVISA) e dá outras providências. Disponível

em:

http://bvsms.saude.gov.br/bvs/saudelegis/anvisa /2010/res0010_09_03_2010.html.

17. Bitocchi, E. et al. Beans (Phaseolus ssp.) as a model for understanding crop evolution. Frontiers in Plant Science, v. 8, p.21, 8 may 2017.

18. Hayat I., Ahmad A., Masud T., Ahmed A., Bashir S. Nutritional and health perspectives of beans (Phaseolus vulgaris L.): an overview. Critical Reviews in Food Science and Nutricion. V. 54, n. 5, p. 580-592, 2014.

19. Silva H.T.; Costa A. O. Caracterização Botânica de Espécies Silvestres do Gênero Phaseolus L. (Leguminosae). Empresa Brasileira de Pesquisa Agropecuária (EMBRAPA). Documentos 156. 2003.

20. Campos-Vega R., Dave Oomah B., Guadalupe Loarca-Piña G., Vergara-Castañeda H. A. Common beans and their non-digestible fraction: Cancer inhibitory activity - an overview. Foods, v. 2, p. 374-392, 2 Aug 2013.

21. Mazur C. E. Efeitos do feijão branco (Phaseolus vulgaris L.) na perda de peso. Revista Brasileira de Nutrição Esportiva, São Paulo. v. 8, n. 48, p.404 - 411. nov./dez. 2014.

22. Montoya C. A., Lallès J.-P., Beebe S., Leterme $\mathrm{P}$. Phaseolin diversity as a possible strategy to improve the nutritional value of common beans (Phaseolus vulgaris). Food Research International, v. 43, p. 443 - 449, 2010.

23. Oliveira A.P., Andrade G. F., Mateó B.S. O., Naozuka J. Protein and metalloprotein distribution in different varieties of beans (Phaseolus vulgaris L.): Effects of cooking. International Journal of Food Science, p. 8, 23 feb 2017.

24. Barrett, M. L.; Udani, J. K. A proprietary alpha-amylase inhibitor from white bean (Phaseolus vulgaris): A review of clinical studies on weight loss and glycemic control. Nutrition Journal, v. 17, p. 10-24, 2011.
25. Celleno L. , Tolaini M.V., D'Amore A., Perricone N.V., Harry G Preuss H.G. A dietary supplement containing standardized Phaseolus vulgaris extract influences body composition of overweight men and women. International Journal of Medical Sciences, v. 1, n. 4, p. 45-52, 2007.

26. Sales P.M., Souza P.M., Simeoni L.A., Silveira D. $\alpha$-Amylase inhibitors: a review of raw material and isolated compounds from plant source. Journal of Pharmacy \& Pharmaceutical Sciences: A Publication of the Canadian Society for Pharmaceutical Sciences, Societe Canadienne Des Sciences Pharmaceutiques, v. 15, n. 1, p. 141-183, 2012.

27. Obiro W. C., Zhang, T., Jiang,B. The nutraceutical role of the Phaseolus vulgaris aamylase inhibitor. British Journal of Nutrition, n.100, p. 1-12, 11 march 2008.

28. Campos-Vega, R., Vergara-Castañeda, A. H., Oomah, B. D. Functional food sources: Beans in sight. Nova Science Publisher, p. 56, New York, United States, 2012.

29. Pavanelli, F. M., Meza, L. K. S., Tiyo, R. Fitoterápicos mais prescritos por nutricionistas de um município paranaense, 2011. Disponível em:

http://www.cesumar.br/prppge/pesquisa/epcc2 011/anais/sheila_karina_luders_meza(1).pdf.

Acesso em: 08 dez 2017.

30. Udani, J., Hardy, M., Madsen, D. C. Blocking carbohydrate absorption and weight loss: a clinical trial using Phase 2 brand proprietary fractionated white bean extract. Alternative Medicine Review, v. 9, n. 1, p. 63-69, mar 2004.

31. Wu X., Xu X., Shen J., Perricone N.V., Preuss H.G. Enhanced Weight Loss From a Dietary Supplement Containing Standardized Phaseolus vulgaris Extract in Overweight Men and Women. The Journal of Applied Research, v.10. n. 2, p. 73-7, 2010.

32. Berkowitz J. Is white bean extract a safe and effective treatment for weight loss? Philadelphia College of Osteopathic Medicine, v.1, n.1, p.1-13, 2011.

33. Onakpoya I., Aldaas S., Terry R., Ernst E. The efficacy of Phaseolus vulgaris as a weightloss supplement: a systematic review and metaanalysis of randomised clinical trials. British Journal of Nutrition., v. 106. N. 2, p. 196-202, 2011. 
34. Embrafarma Pharmaceutical Expertise.

Faseolamina. Disponível em:

http://www.embrafarma.com.br/wpcontent/upl

oads/2017/11/Faseolamina.pdf. Acesso em: 08 dez 2017.

\section{Endereço para Correspondência}

Curso de Pós-graduação Fitoterapia em Nutrição Clínica, Centro Universitário do Triângulo UNITRI

Avenida Nicomedes Alves dos Santos, 4545.

Uberlândia, MG,

CEP.: 38411-106

mariaclarafnunes@outlook.com

Recebido em 24/04/2019

Aprovado em 04/03/2021

Publicado em 31/03/2021 